\title{
Qualia qua qualitons: mental qualities as abstract particulars
}

\begin{abstract}
In this paper we advocate the thesis that qualia are tropes (or qualitons), and not (universal) properties. The main advantage of the thesis is that we can accept both the Wittgensteinian and Sellarsian assault on the given and the claim that only subjective and private states can do justice to the qualitative character of experience. We hint that if we take qualia to be tropes, we dissolve the problem of inverted qualia. We develop an account of sensory concept acquisition that takes the presence of qualia as an enabling condition for learning. We argue that qualia taken to be qualitons are part of our mechanism of sensory concept acquisition.
\end{abstract}

Keywords: qualia, tropes, mental states, sensory vocabulary, concept acquisition

\section{Qualia and (some) of their discontents}

Many people consider plausible that there must be qualitative element to sensory experience that cannot be fully understood in terms of anything like beliefs, disposition to behave or mere neuron firings. These qualitative elements - called qualia - account for the distinctive sensations of cold connected to the water in a mountain stream, of the overwhelming blueness of the deep ocean, of the acute pain associated to cutting oneself in a sharp stone etc. The case for qualia is grounded on the claim that there is something subjective that remains unexplained no matter how you account for the mind in terms of beliefs and conceptual capacities, dispositions to behave or neuron interactions. Qualia-friends hold that any attempt to provide an ontology of the mind should make room for those subjective, qualitative elements. Qualia should therefore be accepted, they conclude, unless we can find resources somewhere else to fully explain them away.

Now, there is little agreement about what sensory experience consists in, and therefore what exactly are qualia (cf. Crane 2008, Gertler, 2008). We believe qualia are interesting if they are taken to have these two related but distinctive features:

i. They are private. That is, they cannot be felt by anybody else but by the person who entertains them. So they are, in an important sense, subjective. 
ii. They are indifferent to beliefs and arguably to other propositional attitudes. Conceptual change cannot affect them. I can find out that there was no dagger in front of me and stop believing there is one, but that cannot rid me of the quale of a dagger in front of me. (This feature can eventually be used to explain illusion situations - like the Müller-Lyer illusion ${ }^{1}$ - where we cannot stop ourselves from seeing what we know is not the case.)

The first feature points at the contrast between public concepts and externally observable behavior on the one hand and states that seem to be only accessible through introspection. The second feature indicates why qualia are generally taken to be inborn or at least non-acquired through conceptual education; they come the same way as heartbeat, blood circulation or muscle flexibility. Still, it is conceivable that one can enhance one's capacity to have qualia, for example, by learning skills (like dancing). Both features together entail that qualia are first-personal and cannot be overruled by third-personal considerations. ${ }^{2}$

David Chalmers has argued that there ought to be room in our ontology for subjective items, independently of their capacity to explain and predict other behavioral or functional states. An ontology informed by future physics (even together with future biology, psychology or sociology) would have to be complemented with the postulation of subjective items like qualia. They are part of the furniture of the universe because they cannot be explained away as the qualitative character of our subjective experience is undeniable (Chalmers 1997). Chalmers is then ready to take the inverted spectrum possibility seriously: two people could correctly identify, say, the same pair of colors but have inverted qualitative experiences associated to each of them. Qualia are conceived as independent of any recognition ability we might have acquired - and still

\footnotetext{
1 Take two parallel lines of the same size. On the top one, place inward arrows in both ends of the line. On the bottom line, place outward arrows in both ends of the line. The bottom one will seem longer.

${ }^{2}$ Several other features commonly attributed to qualia. Typically, for instance, they are taken to be categorical, i.e. non-dispositional states. Different from functional properties, such as a belief understood as what would someone answer if the question arises, qualia have none of this what-wouldbe-if element. Interestingly, one could argue that qualia are dispositional if they are taken to be a disposition to recognize something as something under the right stimuli conditions. Robb \& Heil (2003) diagnose that some of the difficulties around the notion of qualia can be dispelled if we abandon the idea that properties are either dispositional or categorical.
} 
are individuated in terms of our (color) vocabulary. Further, Chalmers also takes seriously the possibility of an particular quale (for instance, the red quale) to be absent. ${ }^{3}$ This appeal to a ready-made qualitative state corresponding to bits of our sensory vocabulary has been met with some suspicion. Non-acquired states seem incapable to carve our sensory vocabulary at its joints - there cannot be any private (sensory) state ready to be labeled by our sensory vocabulary, or so runs the suspicion. In the 20th century, Wittgenstein and Sellars have championed variations of this suspicion: the former argued that there is no such thing as private states that can be identified and individuated without the help of our conceptual practices while the latter was convinced that psychological states could not display primacy with respect to our (conceptually and theoretically loaded) sensory vocabulary. Sellars puts forward what he called psychological nominalism, a doctrine according to which our mental life is made of particulars; in his own words, "all awareness of sorts, resemblances, fatcs etc., in short, all awareness of abstract entities - indeed, all awareness even of particulars - is a linguistic affair" (Sellars 1991, p. 160).

The suspicion has driven many people to recoil from the postulation of qualia. Those subjective, private, incorrigible and non-acquired states seemed implausible on the light either of the criticisms of the plausibility of conceptually unaided sensory discrimination or the mistrust of ready-made sensory items. We will refer to this suspicion as the assault on the given. If one is nevertheless also convinced of Chalmers' motivation to embrace a robust ontology of qualia, one finds oneself swinging between an acceptance of those subjective states on the one hand and a rejection of those states on the other. If one is sensitive to both Chalmers' motivation and Wittgenstein and Sellars' suspicion, a dilemma presents itself: either there are qualia and something is given or nothing is given but the subjective character of our sensory experience remains unexplained. In what follows, we will sketch a way out of the dilemma.

\section{Tropes}

\footnotetext{
${ }^{3}$ Mary, in Jackson thought experiment, is to be understood as having the qualitative counterpart of red missing while she is in the black and white room. As she was not exposed to anything red, she did not display the ready-made expected subjective state corresponding to the image of something red (Jackson 1986). Jackson's Knowledge Argument - there are facts about qualia unknown to Mary in the black and white room - is often used to support the existence of phenomenal concepts, concepts which refer to intrinsic, qualitative properties of experience (Fürst 2004). So the phenomenal concept of 'red' refers to red qualia.
} 
Qualia are often thought of as being qualitative (universal) properties of experience: the red quale can occur in different experiences (and in different minds). (Universal) properties contrast with tropes. Tropes are abstract particulars. While vindicating the nominalist dislike of universals (and properties), the introduction of tropes in an ontology makes room for a conception of objects (concrete particulars) as a collection of abstract particulars. Trope theory can understand both objects and properties as made of the same stuff. Both are made of abstract particulars, such as the (abstract) redness of a particular flower (Campbell 1990).

There could be one-place or many-places tropes; the former are sometimes called qualitons and the latter relatons (see Bacon 2008). Abstract particulars are bound together by some co-presence relation (that could also be a provided by a relaton) forming objects. Instead of properties, trope theory takes every predication to involve particularity; ' $\mathrm{x}$ is a book' doesn't predicate the same property as 'y is a book' - our predicate 'book' does no more than point at some relevant similarity between $\mathrm{x}$ and $\mathrm{y}$; it names no property. The green of a leaf of grass is not the same as the green of another leaf - only they can be relevantly similar, similar enough to be under the same predication.

A trope ontology typically involves tropes (that compose objects), relations of copresence and relations of similarity. In order to provide truth-makers to predications of the form $S$ is $P$, we need those relations of similarity. Similarity relations are often taken to be primary items in trope ontologies. Abstract universals are replaced by abstract particulars and relations of similarity between them. Notice, however, that one can have an ontology with tropes but without those relations of similarity.

Moreover, one could postulate tropes alongside with properties. One could have mixed ontologies where there are abstract universals (and surely there could also be room for concrete particulars irreducible to abstract ones). One could, for example, understand second-order abstractions to be tropes - objects can be predicated with properties while properties could be predicated with tropes. Or one could understand that there are levels of (ontological) similarity: if two tropes are similar enough, they are tokens of the same property. In any case, there could be room for both tropes and (universal) properties.

\section{Qualia as qualitons}

Qualia can be conceived independently of what individuates items in our sensory vocabulary. Michael Tye has recently hinted in this direction. He has argued that there is 
no such thing as a quale corresponding to each state of the world; for example, a quale corresponding to every shade of blue someone is capable to detect (Tye 2006). Tye argues that when two observers disagree as to whether a shade is true blue or greenish blue, there might not be a matter of fact as to which quale is the correct one. It is enough for our species sound adaptation that we have a state corresponding to a reasonablysized class of shades of blue. He's suggesting an independence between an effective and well-adapted sensory state and the qualia that support them. As far as adaptation (or adequacy to detect what is relevant in the surrounding environment) is concerned, the final sensory state is what matters; the (different) qualia being no more than enabling conditions to the this final sensory state.

Jonathan Lowe (1998: 205) presented an argument from perception for the existence of tropes. He claims that both the phenomenology of perceiving changes in (say) the visual features of objects and the acceptance of a causal theory of perception entail that the perceived greenness of a leaf is a particular. A controversy concerning whether tropes are really required in perception followed (see Levinson 2006, Lowe 2008). We shall not present a direct argument in favor of the existence of tropes but rather show the advantages of making room for them in the mind. ${ }^{4}$

We are now in a position to suggest that the dilemma in section 1 was due to an attachment to a metaphysics of properties. If we take qualia to be tropes - no matter whether there are also (universal) properties in the world - we can bring together the advantages of both horns. On the one hand, we can do justice to Chalmers' motivations to postulate a robust ontology of qualia as we consider our sensory states as private, subjective, incorrigible by our beliefs and non-acquired in conceptual education ( $i$ and ii above). On the other hand, we can reject the given together with Wittgenstein and Sellars as there is no need to postulate conceptual contents already present in our qualitative sensory states.

Consider the first horn first. Qualia are abstract particulars that are unaffected by our conceptual tools and indifferent to the uses we make of them to recognize final (conceptually loaded) sensory states like 'green', 'painful' or 'book-shaped'. Our sensory concepts cluster our qualia together but they themselves correspond to no basic qualia qualia are no more than the raw material each of us use to acquire sensory concepts.

\footnotetext{
${ }^{4}$ We think Lowe's argument has to assume too much concerning the phenomenology of perception. Further, it is unclear that in his general terms the universalist (such as Levinson) doesn't have a cogent reply available. Our argument in what follows concentrates rather on how to go about the qualia dilemma we presented in section 1.
} 
Consider the second horn now. Qualia, that are qualitons and not properties, are particulars - just as would be recommended by psychological nominalism. They carry no general content; for instance, they are not subject to Evans' generality constraint. ${ }^{5}$ Hence, it makes scarce sense to talk about inverted qualia. The thought experiments intending to show that qualia can be inverted ask us to think of the different qualia being present in different people in front of the same object. Hence, ripe tomatoes can produce visual qualia in me that are produced by cucumbers in you. The assumption is that a single quale can have multiple occurrences - the same quale being present in one person in front of a tomato and in another in front of a cucumber. If qualia are particulars, we cannot talk of a green or a red quale. So there cannot be tokens of different qualia being prompted by the same object (say a ripe tomato) in different people, as qualia are always different. What corresponds to one person's final sensory state of seeing something red is an array of qualitons, each of them unique. Mary, in Jackson's famous thought experiment (1986), would then be endowed with new qualia when she leaves the black and white room. This means that she now has more resources to recognize something as red. She will now have more qualia, but nothing that can be called a red quale. Therefore, she is not, straight away, in a better position to recognize red objects ${ }^{6}$. A qualiton account of qualia should also say something about similarities between sensory tropes. In order to accommodate our answer to the Wittgenstein-Sellars suspicion concerning the given, we take the relevant similarity relations to be the ones acquired in our sensory concept acquisition. This makes room for Sellars' intuition that there cannot be a ready-made notion of, say, green before we acquire the means to make color predications. There is no non-acquired (or given) way to cluster our qualia together. We cluster them according to the sensory vocabulary we have acquired. The acquisition of sensory concepts is what makes room for patterns of similarity between

\footnotetext{
${ }^{5}$ If we can think of $a$ as an $F a$, there should be at least one $G$ such that we are able to think of $G a$ and at least on $b$ such that we are able to think of $F b$, in order to satisfy the generality constraint (Evans 1982, p. $100-1)$.

${ }^{6}$ Some physicalists deal with Jackson's Knowledge Argument by appealing to Fregean senses. What Mary obtains straight away when she leaves the room is not knowledge of new, irreducible phenomenal facts, but a new mode of presentation of (known) physical facts. This strategy avoids commitment to a Given, but brings in the somewhat mysterious Fregean senses into the picture. Moreover, if senses are part of the world, Mary would then fail to know all the facts before leaving the room. Like Derek Ball (2009), we think it is more promising to dispense with phenomenal concepts altogether. Ball refers to the phenomenon of deference to explain how Mary, still in the room, can possess all color's concepts without having to appeal to phenomenal concepts. So when Mary leaves the room, there is no physical fact new to her. However, Ball does not explain what happens with Mary, what changes take place in her subjective and private life. If qualia are seen as qualitons, Mary simply has new qualitons when she leaves the room. In our approach, as qualitons have no intentional content, nothing would in principle preclude naturalizing qualia.
} 
qualia tropes. Surely, there could be relations of similarity between qualia tropes. ${ }^{7}$ There is no reason to suppose, however, that those existing relations of similarity are either of any relevance for our conceptual acquisition or given to us.

Qualia, but not given relations of similarity, are resources available to us in order to learn how to use the sensory vocabulary that is inculcated in us when we acquire a public language. They enable our learning of a public language together with the relevant correction rules. Qualia are materials for us to exploit while acquiring concepts. Each case of concept acquisition is therefore different from any other. My capacity to recognize something as green depends on my available qualia, and those can differ completely from the qualia anybody else has learned to use to recognize something as green. From those specific qualia, each one builds up resources of recognition exploiting specific relations of similarity.

\section{Sensations, similarities and causal powers}

Let us consider now some implications of seeing qualia as qualitons. If we consider solely the epistemology of sensations - how they justify our beliefs and how they respond to what there is - we feel no need to appeal to qualia. Nor can qualia help, on our account, to explain how our sensations can be adaptive. In this sense, because qualia are only clustered together in stable ways after sensory concepts acquisition, the present account differs from other such as Tye's. The import of qualia for our cognitive life lies in their capacity to support concept acquisition. They enable our capacities of recognition that are fully in place only when a public language is acquired. It provides a model of what goes on privately while a public language is acquired.

In section 304 of the Investigations (1973), Wittgenstein responds to his interlocutor who asks him "But you will surely admit that there is a difference between painbehaviour accompanied by pain and pain-behaviour without any pain? (...) And yet you again and again reach the conclusion that the sensation itself is a nothing". The charge is

\footnotetext{
${ }^{7}$ Qualia as tropes could be part of a naturalized picture of the mind, where those other relations of similarity play a role in our neurophysiology. Michael Beaton (2009) has coined the term moderate phenomenal realism to describe his position in contrast with Chalmers (who would hold a strong version of phenomenal realism). Beaton proposes to define qualia as the introspectible properties (if any) which can still vary (within or between agents), however many properties that can be understood in terms of propositional attitude have been fixed. His proposal, that is still committed to qualia as universals (properties), would constitute a moderate phenomenal realism because it has no commitment to a nonreductive nature of qualia. We believe our proposal would also be in the moderate phenomenal realist camp as qualitons (and relations of similarity between them) could be part of a naturalized picture of the mind.
} 
that our basic qualitative sensory states such as the ones present when we have pain are irrelevant and can be dismissed - it is as if they were not present. Wittgenstein seems to want to say that indeed it is as if they were not present - at least if we describe our mental life in terms of the outcomes of a normal and functioning sensory apparatus but still there is something present. He responds: "Not at all. It is not a something, but it is not a nothing either! The conclusion was only that a nothing would serve just as well as a something about which nothing can be said." The sensation, it seems, appears as a presence that cannot be used unaided in any predication. Yet, relevant relations of similarity are introduced in our mental life with our concept acquisition and when we reach this point, it seems like they can do the job unaided by any sensation. Wittgenstein, then, would be suggesting not that there are no qualia, but rather that there could be qualia if their existence doesn't imply that they are used unaided in predications. It seems as if he is hinting in the direction of taking qualia as tropes. ${ }^{8}$

Our view is therefore one where similarity relations are crucial for the acquisition of concepts. One could, however, fear that judgments of similarity cannot get off the ground if all they have to start out with are mere abstract particulars. Suppose one is learning a sensory concept like 'red' or 'bitter' and has to acquire the relevant similarity relations among her qualia. If one has the quale $\mathrm{Q}$ and is taught that it resembles quale $\mathrm{R}$, but not quale $\mathrm{P}$, how could one compare those qualia without having them somehow present in the mind? In other words, how can my past qualia be retrieved when I need them in order to learn similarity relations if they are not (from the beginning) available in a conceptual format? The question resembles the one Wittgenstein poses at section 342 at the Investigations (1973): how can a deaf-mute person recall thoughts she had before she was introduced to any language, written or otherwise? This is indeed a troublesome area, but we believe we can sketch a way out.

Consider one's attention to quale Q. We assume attention is somehow different from predication - I can attend to Q without making a predication of the sort ' $Q$ is $\varphi$ ' (note that the abstract particular is the subject of the possible predication). If I can attend to Q,

\footnotetext{
${ }^{8}$ Hacker's comments (1993) on this section suggest an interpretation of Wittgenstein which favors, in our view, the view sensations as tropes. He tell us that what Wittgenstein "is doing is rejecting the grammar of name and object". Having a pain is not like to having a penny. So, pain is not concrete. Also, it does not make sense to say that we have now the same pain we have had yesterday. So, pain is not a universal. These together suggest that pain is an abstract particular. At the same time, a sensation is not a nothing, as it's not the absence of anything. It is nothing only in the sense that it cannot be used unaided in predications.
} 
then we can have it present to the mind, at least sometimes, together with P or R. Notice that this procedure of attention can be thoroughly private and subjective - it can differ completely from one to another person. Still, we believe this privacy is both enough to make sure that qualia as qualitons are useful for concept acquisition and does not violate the kernel of the assault on the given. All that is required is that we can manage to attend to two qualitons at the same time so that we can start to grasp the notions of similarity and relevant difference. This should be eventually enough to get the process off the ground - a process of gradual refinement of concepts so that what is roughly red eventually gets refined into different shades of red.

Qualia act as enabling conditions for concept acquisition. They act along with several other states and capacities - among them the capacity to attend to particulars in the world. Our final sensory state will require concepts and we should judge their adaptiveness irrespective of the qualia we have used to acquire them. As mental particulars, qualia are private in the same sense as our genes, our bodies or our voice is private. They are not something more conceptually accessible to me than to anybody else. Yet, they are subjective and satisfy the first feature i above. They are particulars that are subsumed by (sensory) concepts when we have acquired them; they do justice to the intuition that sensations are there to be predicated by our concepts. They are indifferent to our beliefs and hence they also satisfy feature ii - but with a qualification. They cannot by themselves explain illusions (such as the Müller-Lyer illusion mentioned above) as they cannot be used unaided in predications.

When we say that qualia act as enabling conditions for our concept acquisition, we posit a causal efficacy for those qualitons. There has been much discussion recently on whether tropes can solve puzzles concerning mental causation (Robb 1997, Nordhoof 1998, Robb 2003, Heil 2008, Gozzano 2008). Consider Noordhoof's example first. A glass is shattered as a result of a soprano singing a note. It seems tempting to say that it is the pitch and not the meaning of the singing that caused the shattering. Similarly, my feeling of pain (and not the activation of my C-fibers) is what makes me scream. It is in virtue of the pain that I scream. Gozzano, for one, holds that tropes ought to be simples, that is, they must be maximally determinate. If this is so, there should be a pitch-trope and a meaning-trope. Similarly, when considering a causal process that could lead to the acquisition of two different concepts (say, red and yellow), one should posit two and not just one trope. The claim is that if a trope is a simple, it cannot carry two causal powers. Robb, on the other hand, holds that causal powers are connected to particulars - it is a 
particular trope that causes the (particular) shattering of the glass. While it could be that it is better to describe the trope as having a high pitch - each trope has several similarity relations with other tropes - it is the trope qua trope that causes the shattering.

Our view of qualia as qualitons, however, is relatively untouched by the controversy. It is not (solely) in virtue of a trope that we acquire a concept. The relevant causal powers are to be found not only in qualia but also on the concept acquisition context around the process (i.e. the norm-inculcating community). If this is so, a single trope can be part of causal processes of concept acquisition for several color concepts. Further, we claim that a qualia cannot be individuated on the basis of concepts for if it were so, it would fail to escape from the Wittgenstein-Sellars assault on the given. In our account, qualia are enabling conditions for concept acquisition; a given qualiton is neither sufficient nor necessary for a concept to be acquired. Only together with a norm-inculcating community its causal power becomes effective.

\section{Concluding Remarks}

We have put forward a solution for a dilemma concerning qualia. Either we posit them and embrace a form of the given or we reject them and leave bits of our mental life unexplained. If we take them to be tropes instead of (universal) properties, we can have the best of both horns: we can make room for qualia without postulating conceptual contents present in qualitative sensory states. We have sketched an account of concept acquisition where qualia are enabling conditions whose causal powers are triggered only in the context of a norm-inculcating community. As such, qualia as qualitons play no role in the justification of perceptual beliefs.

The thesis that qualia are qualitons is a thesis about our mental life while we acquire and manage sensory concepts. It has no general implications for our ontology of properties or other abstractions. But it has implications for our intuitions concerning sensations and their relations to concepts.

\section{References}

Beaton, M. (2009). Qualia and introspection. Journal of Consciousness Studies 16(5): 88-110.

Bacon, J. (2008). 'Tropes'. The Stanford Encyclopedia of Philosophy (Fall 2008 Edition). E. N. Zalta (ed.). URL = <http://plato.stanford.edu/entries/tropes/>. 
Ball, D. (2009). There Are No Phenomenal Concepts. Mind, 118 (472), 935-962.

Campbell, K. (1990). Abstract Particulars. Oxford: Basil Blackwell.

Chalmers, D. (1997). The Conscious Mind: In Search of a Fundamental Theory. Oxford University Press.

Crane, T. (2008). 'The Problem of Perception'. The Stanford Encyclopedia of Philosophy (Fall 2008 Edition). E. N. Zalta (ed.). URL = <http://plato.stanford.edu/entries/perception-problem/>.

Evans, G. (1982). The Varieties of Reference. Oxford: Oxford University Press.

Fürst, M. (2004). Qualia and Phenomenal Concepts as Basis of the Knowledge Argument. Acta Analytica, 19(32), 143-152.

Gozzano, S. (2008). Tropes’simmplicity and mental causation. In: Gozano \& Orilia (2008), 133-154.

Gozzano, S. \& Orilia, F., eds. (2008). Tropes, Universals and the Philosophy of Mind, Frankfurt: Ontos-Verlag.

Gertler, B. (2008). 'Self-Knowledge'. The Stanford Encyclopedia of Philosophy, (Winter 2008 Edition). $\quad$ E. N. Zalta (ed.). URL = < http://plato.stanford.edu/entries/self-knowledge/>.

Hacker, P. M. S. (1993). Wittgenstein: Meaning and Mind, Volume 3 of an Analytical Commentary on the Philosophical Investigations, Part II: Exegesis 243-42. Wiley-Blackwell.

Heil, J. (2008). Modes and mind. In: Gozzano \& Orilia (2008), 13-30.

Jackson, F. (1986). What Mary Didn't Know. The Journal of Philosophy, 85(5), 291295.

Levinson, J. (2006). Why there are no tropes. Philosophy. 81, 563-579.

Lowe, J. (1998). The Possibility of Metaphysics: Substance, Identity and Time. Oxford: Oxford: Clarendon Press

Lowe, J. (2008). Tropes and perception. In: Gozzano \& Orilia (2008), pp. 175-194.

Nordhoof, P. (1998). Do tropes resolve the problem of mental causation? The Philosophical Quarterly, 48, 221-226.

Robb, J. (1997). The problems of mental causation. Philosophical Quarterly, 47, 178194.

Robb, D. (2003). Reply to Noordhof on Mental Causation. The Philosophical Quarterly, 51, 202, 90-94.

Robb, D., \& Heil, J. (2003). Mental Properties. American Philosophical Quarterly, 
40(3), 175-196.

Sellars, W. (1991). Science, Perception and Reality. Ridgeview Publishing Company.

Tye, M. (2006). The Puzzle of True Blue. Analysis, 66(4), 340-344.

Wittgenstein, L. (1973). Philosophical Investigations, Oxford, Blackwell. 